\title{
EQUIVARIANT CW COMPLEXES AND SHAPE THEORY
}

Dedicated to Professor Masahiro Sugawara on his 60th birthday

\author{
By
}

\section{Takao Matumoto}

The aim of this note is to study a discrete group equivariant shape theory by associating an inverse system in the homotopy category of equivariant $\mathrm{CW}$ complexes.

\section{Introduction}

Let $G$ be a discrete group and $X$ a $G$-space. For a subgroup $H$ of $G$ we denote $X^{H}=\{x \in X ; g x=x$ for every $g \in H\}$. For a $G$-map $f: X \rightarrow Y$ of $X$ to another $G$-space $Y$, we denote $f^{H}=f \mid X^{H}: X^{H} \rightarrow Y^{H}$. Let $\mathscr{H}_{G}$ denote the category of $G$-spaces and $G$-homotopy classes of $G$-maps and $\mathscr{W}_{G}$ the full subcategory of $\mathscr{H}_{G}$ consisting of $G$-spaces which have the $G$-homotopy types of $G$-CW complexes.

THEOREM 1. There is a functor $\check{C}_{G}$ from $\mathscr{H}_{G}$ into the pro-category pro- $\mathscr{W}_{G}$ of $\mathscr{W}_{G}$ so that $\check{C}_{G}(X)=\left(X_{\lambda},\left[p_{\lambda \lambda^{\prime}}^{X}\right]_{G}, \Lambda\right)$ has the universal property for the equivariant shape theory with a system G-map $p^{X}=\left(\left[p_{\lambda}^{X}\right]_{G}\right): X \rightarrow \check{G}_{G}(X)$, that is, $p^{X}: X \rightarrow \check{C}_{G}(X)$ is a G-CW expansion of $X$.

When $G$ is a finite group, we know that a $G$-ANR has the $G$-homotopy type of a $G-C W$ complex and vice versa. Also any numerable covering has a refinement of numerable $G$-equivariant covering. So, we have

TNEOREM 2. Let $G$ be a finite group and $X$ a G-space.

(1) Any G-ANR expansion of $X$ is equivalent to $p^{X}: X \rightarrow \check{C}_{G}(X)$.

(2) The expansion $p^{X}: X \rightarrow \check{C}_{G}(X)$ is a (non-equivariant) $\mathrm{CW}$ expansion of $X$. Moreover, if $X$ is a normal G-space, then $p^{X, H}=\left(\left[p_{\lambda}^{X}, H\right]\right): X^{H} \rightarrow \check{C}_{G}(X)^{H}=$ $\left(X_{\lambda}^{H},\left[p_{\lambda \lambda^{\prime}}^{X}\right], \Lambda\right)$ is a $\mathrm{CW}$ expansion for every subgroup $H$ of $G$.

(3) Let $f: X \rightarrow Y$ be a G-map between normal $G$-spaces. Then, $\check{C}_{G}(f): \check{G}_{G}(X)$ $\rightarrow \check{C}_{G}(Y)$ is an isomorphism in pro- $\mathscr{W}_{G}$ if and only if $f^{H}: X^{H} \rightarrow Y^{H}$ is a shape

Received January 25, 1988. Revised June 6, 1988. 
equivalence for every subgroup $H$ of $G$.

The case when $G$ is a finite group is also treated by Pop [10]. But he did not mention on (2) and (3) of Theorem 2. We note also that Antonian-Mardešić [1] defined the equivariant ANR shape for compact groups. Our treatment in the case when $G$ is not a discrete group will be discussed elsewhere.

\section{A quick review of shape theory}

The general references are [3], [4] and [8]. Borsuk (1968) defined the shape for compact metric spaces, Mardešić-Segal (1971) for compact Hausdorff spaces, Fox (1972) for metric spaces, and Mardešić (1973) and K. Morita (1975) for topological spaces.

Let $\boldsymbol{X}=\left(X_{\lambda}, p_{\lambda \lambda^{\prime}}, \Lambda\right)$ and $\boldsymbol{Y}=\left(Y_{\mu}, q_{\mu \mu^{\prime}}, M\right)$ be inverse systems in a category c. A system map of $\boldsymbol{X}$ to $\boldsymbol{Y}$ consists of $\theta: M \rightarrow \Lambda$ and morphisms $f_{\mu}: X_{\theta(\mu)}$ $\rightarrow Y_{\mu}$ in $\mathcal{C}$ satisfying $q_{\mu \mu^{\prime}} f_{\mu^{\prime}} p_{\theta\left(\mu^{\prime}\right) \lambda}=f_{\mu} p_{\theta(\mu) \lambda}$ for $\mu \leqq \mu^{\prime}, \theta\left(\mu^{\prime}\right) \leqq \lambda$ and $\theta(\mu) \leqq \lambda$. Two system maps $\left(f_{\mu}, \theta\right)$ and $\left(f_{\mu}^{\prime}, \theta^{\prime}\right)$ are said to be equivalent if each $\mu \in M$ admits a $\lambda \in \Lambda, \lambda \geqq \theta(\mu)$ and $\lambda \geqq \theta^{\prime}(\mu)$, such that $f_{\mu} p_{\theta(\mu) \lambda}=f_{\mu}^{\prime} p_{\theta^{\prime}(\mu) \lambda}$. The procategory pro- $\mathcal{C}$ of the category $\mathcal{C}$ is defined by $\mathrm{Obj}$ (pro- $\mathcal{C}$ )=all inverse systems in $\mathcal{C}$ and $\operatorname{Mor}(\boldsymbol{X}, \boldsymbol{Y})=$ equivalence classes of system maps of $\boldsymbol{X}$ to $\boldsymbol{Y}$. Let $\mathscr{D}$ be a full subcategory of $\mathcal{C}$. A $\mathscr{D}$-expansion $p=\left(p_{\lambda}\right): X \rightarrow X$ of $X$ is a system map which is characterized by the following universal properties due to Mardešić $[4$, Ch. I, Th. I]:

(0) $X_{\lambda} \in \mathscr{D}$ for each $\lambda \in \Lambda$.

(1) For any map $f: X \rightarrow K$ with $K \in \mathscr{D}$ there exists a morphism $h_{\lambda}: X_{\lambda} \rightarrow K$ such that $f=h_{\lambda} p_{\lambda}$.

(2) If $f=g_{\lambda} p_{\lambda}$ then there is a $\lambda^{\prime} \geqq \lambda$ such that $h_{\lambda} p_{\lambda \lambda^{\prime}}=g_{\lambda} p_{\lambda \lambda^{\prime}}$.

We give an exact definition of Čech expansion and Čech system due to Morita. Let $\mathscr{W}$ be the homotopy category of spaces which have homotopy type of $\mathrm{CW}$ complexes.

For a space $X$ we associate an inverse system $\check{C}(X)=\left(X_{\lambda},\left[p_{\lambda \lambda^{\prime}}^{X}\right], \Lambda\right)$ in $\mathscr{W}$ by

$$
\begin{aligned}
& \left\{\mho_{\lambda}\right\}_{\lambda \in \Lambda}=\text { all numerable coverings of } X, \lambda^{\prime} \geqq \lambda \text { iff } \mathscr{U}_{\lambda^{\prime}}\left\langle\mathcal{U}_{\lambda} ;\right. \\
& X_{\lambda}=N\left(\mathcal{U}_{\lambda}\right) \text { and } p_{\lambda \lambda^{\prime}}^{X}: N\left(\mathcal{U}_{\lambda^{\prime}}\right) \rightarrow N\left(\mathcal{U}_{\lambda}\right),
\end{aligned}
$$

where $N\left(U_{\lambda}\right)$ is the nerve of $\mathcal{U}_{\lambda}=\left\{U_{\alpha}^{\lambda}\right\}$ and $p_{\lambda \lambda^{\prime}}^{X}$ is a simplicial map defined by choosing $\tilde{p}=p_{\lambda \lambda^{\prime}}^{X}$ so that $U_{\alpha}^{\lambda^{\prime}} \subset U_{\tilde{p}(\alpha)}^{\lambda}$. The homotopy class $\left[p_{\lambda \lambda^{\prime}}^{X}\right]$ is independent of the choice of $\tilde{p}$. Then the inverse system $\check{C}(X)$ in pro- $\mathscr{W}$ well-defined and 
is called the Čech system of $X$. Here a pointwise finite covering $U=\left\{U_{\alpha}\right\}$ of $X$ is called numerable if it admits a locally finite partition of unity $\left\{\rho_{\alpha}\right\}$ i. e., a family of continuous functions $\rho_{\alpha}: X \rightarrow[0,1]$ with $\Sigma \rho_{\alpha}=1$ and $\rho_{\alpha}^{-1}(0,1] \subset U_{\alpha}$ such that $\left\{\rho_{\alpha}^{-1}(0,1]\right\}$ is a locally finite covering of $X$. By the locally finite partition of unity $\left\{\rho_{\alpha}\right\}$ subordinate to $U_{\lambda}$ we have a map $p_{\lambda}^{X}: X \rightarrow X_{\lambda}$ defined by $p_{\lambda}^{X}(x)=\Sigma \rho_{\alpha}(x)\left\langle U_{\alpha}\right\rangle$ where $\left\langle U_{\alpha}\right\rangle \in X_{\lambda}$ is the vertex corresponding to $U_{\alpha}$. A different choice of the locally finite partition of unity gives another map contiguous to $p_{\lambda}^{X}$. So, the homotopy class of $p_{\lambda}^{X}$ depends only on $V_{\lambda}$ and $p_{\lambda \lambda^{\prime}}^{X} p_{\lambda^{\prime}}^{X}$ $\simeq p_{\lambda}^{X}$. Then $p^{X}=\left(\left[p_{\lambda}^{X}\right]\right): X \rightarrow \check{C}(X)$ is a $\mathscr{W}$-expansion and called the Čech expansion of $X$.

Any $\mathscr{W}$-expansion $X \rightarrow X$ is equivalent to the Čech expansion $p^{X}: X \rightarrow \check{C}(X)$. The equivalence class of $\mathscr{W}$-expansion of $X$ is called the shape of $X$.

\section{Equivariant Čech system $\check{C}_{G}(X)$ (Proof of Theorem 1)}

Let $G$ be a discrete group and $X$ a $G$-space. An open covering $U=\left\{U_{\alpha}\right\}$ of $X$ is called a numerable $G$-equivariant covering if $g U_{\alpha}=U_{g \alpha} \in \mathcal{U}$ for each $U_{\alpha} \in \mathcal{U}$ and $g \in G$ and if $\mathcal{U}$ has a locally finite partition of unity $\left\{\rho_{\alpha}\right\}$ such that $\rho_{g \alpha}(x)=\rho_{\alpha}\left(g^{-1} x\right)$ for any $g \in G$ and the following three sets have finite differences :

$$
\left\{g \in G ; g \alpha=\alpha \text { i. e., } \rho_{g \alpha}=\rho_{\alpha}\right\} \subset\left\{g \in G ; g U_{\alpha}=U_{\alpha}\right\} \subset\left\{g \in G ; g U_{\alpha} \cap U_{\alpha} \neq \varnothing\right\} .
$$

The nerves $X_{\lambda}=N\left(\vartheta_{\lambda}\right)$ of the numerable $G$-equivariant coverings $U_{\lambda}$ of $X$ induce an inverse system $\check{C}_{G}(X)=\left(X_{\lambda},\left[p_{\lambda \lambda^{\prime}}^{X}\right]_{G}, \Lambda\right)$ in $\mathscr{W}_{G}$ with a system $G$-map $p^{X}=\left(\left[p_{\lambda}^{X}\right]_{G}: X \rightarrow X_{\lambda}\right)$ such that $p_{\lambda}^{X} \simeq_{G} p_{\lambda \lambda^{\prime}}^{X} p_{\lambda^{\prime}}^{X}$. The $G$-homotopy classes $\left[p_{\lambda}^{X}\right]_{G}$ and $\left[p_{\lambda \lambda^{\prime}}^{X}\right]_{G}$ are also well-defined by the argument using contiguity as in the non-equivariant case.

For a $G$-map $f: X \rightarrow Y$ a system $G$-map $\check{C}_{G}(f)=\left(\left[f_{\mu}\right]_{G}, \theta\right): \check{C}_{G}(X)=$ $\left(X_{\lambda},\left[p_{\lambda \lambda^{\prime}}^{X}\right]_{G}, \Lambda\right) \rightarrow \check{C}_{G}(Y)=\left(Y_{\mu},\left[p_{\mu \mu^{\prime}}^{X}\right]_{G}, M\right)$ is defined so that $p_{\mu}^{Y} f \simeq_{G} f_{\mu} p_{\theta(\mu)}^{X}$. In fact, a numerable $G$-equivariant covering $\mathcal{V}_{\mu}=\left\{V_{\beta}^{\mu}, \rho_{\beta}\right\}$ of $Y$ induces a covering $f^{-1} C V_{\mu}=\left\{f^{-1}\left(V_{\beta}^{\mu}\right), \rho_{\beta} f\right\}$ of $X$, which is numerable $G$-equivariant and may be denoted by $\mho_{\theta(\mu)}$, and $f_{\mu}: N\left(f^{-1} C_{\mu}\right) \rightarrow N\left(\mathcal{V}_{\mu}\right)$ defined by the natural inclusion satisfies the required $G$-homotopy equality.

Hereafter we will omit []$_{G}$ to avoid complexity of notation.

LEMma 3.1. Let $K$ be a G-CW complex. Then, the system G-map $p^{K}: K \rightarrow$ $\check{C}_{G}(K)$ is an isomorphism in pro- $\mathscr{W}_{G}$.

LEMMA 3.2. For a G-space $X$ we take a G-map $p_{\lambda}^{X}: X \rightarrow X_{\lambda}$ in the system 
G-map $p^{X}=\left(p_{\lambda}^{X}\right): X \rightarrow \check{C}_{G}(X)$ and consider a system $G$-map $\check{C}_{G}\left(p_{\lambda}\right)=\left(\left(p_{\lambda}^{X}\right)_{\mu}, \varphi_{\lambda}\right)$ : $\check{C}_{G}(X) \rightarrow \check{C}_{G}\left(X_{\lambda}\right)$. Then, there is a $\nu$ with $\nu \geqq \lambda$ and $\nu \geqq \varphi_{\lambda}(\mu)$ such that $p_{\mu}^{\lambda} p_{\lambda \nu}^{X} \simeq_{G}$ $\left(p_{\lambda}^{X}\right)_{\mu} p_{\varphi_{\lambda}(\mu) \nu}^{X}$, where $p_{\mu}^{\lambda}$ denotes $p_{\mu}^{X \lambda}$.

LEMMA 3.3 (Universal property for equivariant shape). Let $p^{X}=\left(p_{\lambda}^{X}\right): X \rightarrow$ $\check{C}_{G}(X)=\left(X_{\lambda}, p_{\lambda \lambda^{\prime}}^{X}, \Lambda\right)$ be the system $G$-map defined above. Let $K$ be a G-CW complex and $f: X \rightarrow K a G$-map.

(1) There exist $a \lambda$ and a G-map $h: X_{\lambda} \rightarrow K$ such that $f \simeq_{G} h p_{\lambda}^{X}$.

(2) If $f \simeq_{G} g p_{\lambda}^{X}$ for any other $G$-map $g: X_{\lambda} \rightarrow K$, then there is $a \nu$ with $\nu \geqq \lambda$ such that $h p_{\lambda_{2}}^{X} \simeq G g p_{\lambda_{2}}^{X}$.

Proof of Lemma 3.3 AND TheOREM 1 From Lemmas 3.1 and 3.2. Lemma 3.3 is a detailed restatement of Theorem 1. Lemmas 3.1 and 3.2 imply Lemma 3.3 in a standard way. In fact, the system $G$-map $\check{C}_{G}(f): \check{C}_{G}(X) \rightarrow \check{C}_{G}(K)$ consists of $\theta: M \rightarrow \Lambda$ and $G$-maps $f_{\mu}: X_{\theta(\mu)} \rightarrow K_{\mu}$. By Lemma 3.1 we have a $\mu$ and a $G$-map $q: K_{\mu} \rightarrow K$ such that $q p_{\mu}^{K} \simeq_{G} i d_{K}$. Now it suffices to define $\lambda=\theta(\mu)$ and $h=q f_{\mu}$ to prove (1), because $q f_{\mu} p_{\theta(\mu)}^{X} \simeq_{G} q p_{\mu}^{K} f \simeq_{G} f$. To prove (2) we note that $q g_{\mu} p_{\theta_{\lambda}(\mu)} \simeq_{G} g$ replacing $X, f$ and $\theta$ with $X_{\lambda}, g$ and $\theta_{\lambda}$ respectively. By Lemma 3.1 there is a $G$-map $q^{\prime}:\left(X_{\lambda}\right)_{\nu} \rightarrow X_{\lambda}$ with $\nu \geqq \theta_{\lambda}(\mu)$ such that $q^{\prime} p_{\nu}^{\lambda} \simeq_{G} i d$ and $p_{\nu}^{\lambda} q^{\prime} p_{\nu \tilde{\nu}}^{\lambda} \simeq_{G} p_{\nu \tilde{\nu}}^{\lambda}$ for some $\tilde{\nu} \geqq \nu$, where $p_{\nu \nu^{\prime}}^{\lambda}$ denotes $p_{\nu \nu^{\prime}}^{X}$. So, $g q^{\prime} p_{\nu \tilde{\nu}}^{\lambda} \simeq_{G} q g_{\mu} p_{\nu \tilde{\nu}}^{\lambda}$. Here we retake $\theta_{\lambda}(\mu)=\nu$. Take the $G$-map $\left(p_{\lambda}^{X}\right)_{\tilde{\nu}}: X_{\nu^{\prime}} \rightarrow\left(X_{\lambda}\right)_{\tilde{\nu}}$ by putting $\nu^{\prime}=\varphi_{\lambda}(\tilde{\nu})$. Then, since $g p_{\lambda}^{X} \simeq_{G} f$, we have a $\tilde{\nu}^{\prime}$ with $\tilde{\nu}^{\prime} \geqq \nu^{\prime}$ and $\tilde{\nu}^{\prime} \geqq \theta(\mu)$ such that $g_{\mu} p_{\nu \tilde{\nu}}^{\lambda}\left(p_{\lambda}^{X}\right)_{\tilde{\nu}} p_{\nu^{\prime} \tilde{\nu}^{\prime}}^{X} \simeq_{G} f_{\mu} p_{\theta(\mu) \tilde{\nu}^{\prime}}^{X}$. So, $g q^{\prime} p_{\nu \tilde{\nu}}^{\lambda}\left(p_{\lambda}^{X}\right)_{\tilde{\nu}} p_{\nu^{\prime} \tilde{\nu}^{\prime}}^{X} \simeq_{G} q f_{\mu} p_{\theta(\mu) \tilde{\nu}^{\prime}}^{X}$. On the other hand by Lemma 3. 2 we have $p_{\nu \tilde{\nu}}^{\lambda}\left(p_{\lambda}^{X}\right)_{\Sigma} p_{\nu^{\prime} \tilde{\nu}^{\prime}}^{X} \simeq_{G} p_{\nu}^{\lambda} p_{\lambda \tilde{\nu}}^{X}$, if necessary retaking a larger $\tilde{\nu}^{\prime}$. Hence, $g p_{\lambda \tilde{\nu}^{\prime}}^{X} \simeq_{G} q f_{\mu} p_{\theta^{\prime}(\mu) \tilde{\nu}^{\prime}}^{X} \simeq_{G} h p_{\lambda \tilde{\nu}^{\prime}}^{X}$.

q. e. d.

Proof of Lemma 3.1. We consider a natural $G$-map $\sigma:|S(K)| \rightarrow K$ for the geometric realization of the singular complex of $K$. Since $|S(K)|^{H}=\left|S\left(K^{H}\right)\right|$, we see that $\sigma$ is a $G$-homotopy equivalence. Since a $G$-homotopy equivalence induces an isomorphism $\check{C}_{G}(\cdot)$ in pro- $\mathscr{W}_{G}$, the proof reduces to the following two lemmas.

LEMMA 3.4. For a G-space $X,|S(X)|$ admits a G-equivariant triangulation.

LEMMA 3.5. For a G-equivariantly triangulated G-space $K, p^{K}: K \rightarrow \check{G}_{G}(K)$ is an isomorphism in pro- $\mathscr{W}_{G}$. Moreover, suppose $\mu$ is given then there are a $\tilde{\mu}$ $\left(\geqq \mu\right.$ ) and a G-map $q: K_{\tilde{\mu}} \rightarrow K$ such that $q$ is the G-homotopy inverse to $p_{\tilde{\mu}}^{K}$.

Proof of LEMMA 3.4. We know that there is a $G$-homeomorphism between 
$|S(X)|$ and $|\operatorname{Sd} S(X)|$ where $\operatorname{Sd} S(X)$ is a barycentric subdivision of the singular s. s. complex $S(X)$ of $X$. Note that the natural quotient map $|\operatorname{Sd} S(X)| \rightarrow$ $|\mathrm{Sd} S(X) / G|$ restricts to a homeomorphism on any cell of $|\mathrm{Sd} S(X)|$. So, a triangulation of the regular $\mathrm{CW}$ complex $|\operatorname{Sd} S(X) / G|$ lifts to a $G$-equivariant triangulation of $|\operatorname{Sd} S(X)|$.

q.e.d.

PROOF OF LEMMA 3.5. For each vertex $v$ we take an open star neighborhood $U_{v}$. Then, $v_{1}, \cdots, v_{n}$ are the vertices of the same simplex if and only if $U_{v_{1}} \cap \cdots \cap U_{v_{n}}$ is not empty. If necessary by taking a barycentric subdivision, we may assume the following: If $g v$ and $v$ are in the same simplex of $K$ then $g v=v$ and hence $U_{g v} \cap U_{v} \neq \varnothing$ implies $g v=v$. We put $\bar{\rho}_{v}(x)=$ the coefficient of $x$ with respect to $v$. Then the $G$-map $\bar{p}: K \rightarrow N\left(\left\{U_{v}\right\}\right)$ defined by $\left\{\bar{\rho}_{v}\right\}$ is not only a bijection but also a $G$-homeomorphism. Note here that $\bar{\rho}_{v}(g x)=\bar{\rho}_{v}(x)$ if $g v=v$. Now we make the support of $\bar{\rho}_{v}$ smaller and get a locally finite $G$ equivariant partition of unity $\rho_{v}$ so that $\mathcal{U}=\left\{U_{v}, \rho_{v}\right\}$ is a numerable $G$-equivariant covering and $p: K \rightarrow N(\mathcal{U})$, defined by $\left\{\rho_{v}\right\}$, is $G$-homotopic to $\bar{p}: K \rightarrow N(\mathcal{U})$. If we take a subdivision of $K$ fine enough at first, we may assume that $\mho_{\langle}\left\langle\mathcal{V}_{\mu}\right.$. Take this $U$ as $U_{\tilde{\mu}}$. Then $p_{\tilde{\mu}}: K \rightarrow K_{\tilde{\mu}}=N\left(\mathcal{U}_{\tilde{\mu}}\right)$ is a $G$-homotopy equivalence. This finishes the proof of Lemma 3.5 and also Lemma 3.1.

q. e. d.

Proof of Lemma 3.2. Note that $X_{\lambda}$ is equivariantly triangulated. By the proof of Lemma 3.5 we have a $\tilde{\mu}(\geqq \mu)$ and a subdivision $X_{\lambda}^{\prime}$ of $X_{\lambda}$ such that $\bigcup_{\tilde{\mu}}$ is the open star covering of $X_{\lambda}^{\prime}$ and $p_{\tilde{\mu}}^{\lambda}: X_{\lambda}^{\prime} \rightarrow\left(X_{\lambda}\right)_{\tilde{\mu}}=N\left(\mathcal{U}_{\tilde{\mu}}\right)$ is $G$-homotopic to the natural identification. The $G$-map $p_{\lambda}^{X}$ induces a numerable $G$-equivariant covering $\mathcal{U}_{\nu}=\left(p_{\lambda}^{X}\right)^{-1}\left(\mathcal{U}_{\tilde{\mu}}\right)$ of $X$ and the natural inclusion $\left(p_{\lambda}^{X}\right)_{\tilde{\mu}}: X_{\nu}=N\left(\mathcal{U}_{\nu}\right) \rightarrow\left(X_{\lambda}\right)_{\tilde{\mu}}$ $=N\left(\mathcal{U}_{\tilde{\mu}}\right)$. The $G$-map $p_{\lambda \nu}^{X}$ is the composition of the inclusion $X_{\nu} \rightarrow X_{\lambda}^{\prime}$ with a simplicial $G$-map $X_{\lambda}^{\prime} \rightarrow X_{\lambda}$ given by choosing a refinement. Hence $p_{\tilde{\mu}}^{\lambda} p_{\lambda \nu}^{X} \simeq_{G}\left(p_{\lambda}^{X}\right)_{\tilde{\mu}}$. This implies Lemma 3.2 and completes a proof of Theorem 1. q.e.d.

\section{The case when $G$ is a finite group}

Let $G$ be a finite group and $X$ a $G$-space. Then (1) of Theorem 2 is a consequence of Theorem 1 and the fact that a $G$-ANR has the $G$-homotopy of a G-CW complex and vice versa (cf. [9] and [4, Appendix] or [10]). Pop [10] also defines the equivariant shape theory for a finite group $G$. In the case that $X$ is normal, (2) and (3) of Theorem 2 enrich the result.

LEMMA 4.1. Let $G=\left\{g_{1}, \cdots, g_{n}\right\}$ be a finite group. For any numerable covering $U=\left\{U_{\alpha}, \rho_{\alpha}\right\}$ of a G-space $X$ we have a numerable G-equivariant cover- 
ing $\mathscr{V}$ of $X$ such that $\mathcal{V}<U$.

PROOF. It suffices to take the covering $\mathcal{V}$ consisting of $g_{1}^{-1} U_{\alpha_{1}} \cap \cdots \cap g_{n}^{-1} U_{\alpha_{n}}$ with $\rho_{\alpha_{1}}\left(g_{1} x\right) \cdots \rho_{\alpha_{n}}\left(g_{n} x\right)$. In fact, $g_{i}\left(g_{1}^{-1} U_{\alpha_{1}} \cap \cdots \cap g_{n}^{-1} U_{\alpha_{n}}\right) \subset U_{\alpha_{i}}$ and the sum $\sum \rho_{\alpha_{1}}\left(g_{1} x\right) \cdots \rho_{\alpha_{n}}\left(g_{n} x\right)$ is equal to $\left(\sum \rho_{\alpha_{1}}\left(g_{1} x\right)\right) \cdots\left(\sum \rho_{\alpha_{n}}\left(g_{n} x\right)\right)=1$. Note that we do not require $g V_{\beta} \cap V_{\beta} \neq \varnothing$ implies $g V_{\beta}=V_{\beta}$ for the numerable $G$-equivariant covering.

q. e. d.

Proof of (2) OF THEOREM 2. Lemma 4.1 implies that $p^{X}: X \rightarrow \check{C}_{G}(X)$ is also a (non-equivariant) CW expansion of $X[4$, Ch. I, $\S 1$, Th. $1 ; \S 2$, Rem. 3]. Assume that $X$ is a normal space. For a subgroup $H$ of $G$ any numerable covering $U_{H}$ of the closed subspace $X^{H}$ extends to a numerable covering $U$ of $X$ i.e., $U_{H}=\left\{U \cap X^{H} ; U \in U\right\}$. We may assume that if $U \cap X^{H}=\varnothing$ then $U$ is not $H$-invariant for $U \in \mathcal{V}$. So, we see that $\check{C}_{G}(X)^{H} \simeq \check{C}_{W(H)}\left(X^{H}\right)$ for a normal $G$-space $X$ where $W(H)=N(H) / H$ and $N(H)=\left\{g \in G ; g H g^{-1}=H\right\}$. Now we have proved (2) of Theorem 2 by considering $X^{H}$ a $W(H)$-space. q.e.d.

LEMMA 4.2. Let $G$ be a finite group. Let $X$ and $Y$ be $G-C W$ complexes and $h_{H}: X^{H} \rightarrow Y^{H}$ maps satisfying $g_{*} h_{H} \simeq h_{H^{\prime}} g_{*}$ for every pair of subgroups $H^{\prime} \subset g H g^{-1}$ where $g_{*}(x)=g x$. Then there is a G-map $f: X \rightarrow Y$ such that $f \mid X^{H}$ $\simeq h_{H}$ for every subgroup $H$ of $G$.

Proof. Choose a family of representatives $\left\{H_{1}, \cdots, H_{m}\right\}$ of conjugacy classes of subgroups of $G$. For $G$-0-cell $\sigma: \Delta^{0} \times G / H_{i} \rightarrow X$ we define $f \mid X^{0}$ by $f\left(\sigma\left(\Delta^{0} \times g H_{i} / H_{i}\right)\right)=g_{*} h_{H_{i}}\left(\sigma\left(\Delta^{0} \times H_{i} / H_{i}\right)\right)$. Assume that a $G$-map $f \mid X^{n-1}$ is defined and for $H=H_{i}$ there are given homotopies between $f \mid \sigma\left(\Delta^{k} \times H / H\right)$ and $h_{H} \mid \sigma\left(\Delta^{k} \times H / H\right)$ in $Y^{H}$ which extend the homotopies on the boundaries as an induction hypothesis for $k<n$. Then, for a $G$ - $n$-cell $\sigma: \Lambda^{n} \times G / H \rightarrow X$ with $H=H_{i}, h_{H} \mid \sigma\left(\partial \Delta^{n} \times H / H\right)$ is homotopic to $f \mid \sigma\left(\partial \Delta^{n} \times H / H\right)$. We can now define $f \mid \sigma\left(\Delta^{n} \times H / H\right)$ by the homotopy on the collar and by $h_{H}$ on the interior. Extending $f$ on $\sigma\left(\Delta^{n} \times G / H\right)$ so that $f$ becomes $G$-equivariant, $f \mid X^{n}$ satisfies also the induction hypothesis. So, we get a $G$-map $f: X \rightarrow Y$ such that $f \mid X^{H} \simeq h_{H}$.

q.e.d.

Proof of (3) OF THEOREM 2. If $f: X \rightarrow Y$ induces an isomorphism $\check{C}_{G}(f)$ : $\check{C}_{G}(X) \rightarrow \check{C}_{G}(Y)$ in pro- $\mathscr{W}_{G}$, then all $\check{C}_{G}(f)^{H}: \check{C}_{G}(X)^{H} \rightarrow \check{C}_{G}(Y)^{H}$ are isomorphisms in pro- $\mathscr{W}$. This means that all $f^{H}: X^{H} \rightarrow Y^{H}$ are shape equivalences by (2) of Theorem 2. Now suppose that all $f^{H}: X^{H} \rightarrow Y^{H}$ are shape equivalences. Then, also by (2) of Theorem $2, \check{C}_{G}(f)^{H}=\left(f_{\mu}^{H}, \lambda\right): \check{C}_{G}(X)^{H} \rightarrow \check{C}_{G}(Y)^{H}$ are isomorphisms in 


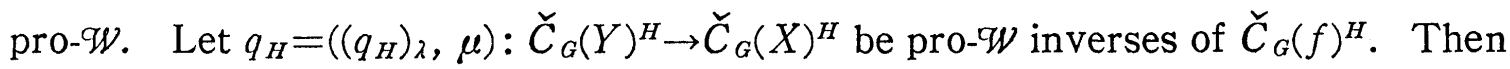
$\left(q_{H}\right)_{\lambda} f_{\mu}^{H} p_{\lambda, \tilde{\lambda}}^{X, H} \simeq p_{\lambda \tilde{\lambda}}^{X, H}$ for some $\tilde{\lambda} \geqq \lambda^{\prime}$ and $f_{\mu}^{H} p_{\lambda, \tilde{\lambda}}^{X, H}\left(q_{H}\right)_{\bar{\lambda}} p_{\mu^{\prime}, \tilde{\mu}}^{Y, H} \simeq p_{\mu \tilde{\mu}}^{Y, H}$ for some $\tilde{\mu} \geqq \mu^{\prime}$. Here we abbreviate $\mu=\mu(\lambda), \lambda^{\prime}=\lambda(\mu)$ and $\mu^{\prime}=\mu(\tilde{\lambda})$. By taking $\mu, \lambda^{\prime}, \tilde{\lambda}, \mu^{\prime}$ and $\tilde{\mu}$ equal to or bigger than the ones for each $H$, we may assume that they do not depend on $H$. Note that if $H^{\prime} \subset g H g^{-1}$ then $g_{*} f_{\mu}^{H} \simeq f_{\mu}^{H^{\prime}} g_{*}, g_{*} p_{\lambda \lambda^{\prime}}^{X} \simeq p_{\lambda \lambda^{\prime}}^{X}{ }^{\prime} g_{*}$ and $g_{*} p_{\mu \mu^{\prime}}^{Y, H} \simeq p_{\mu \mu^{\prime}}^{Y, H^{\prime}} g_{*}$. We have in this case the following diagram:

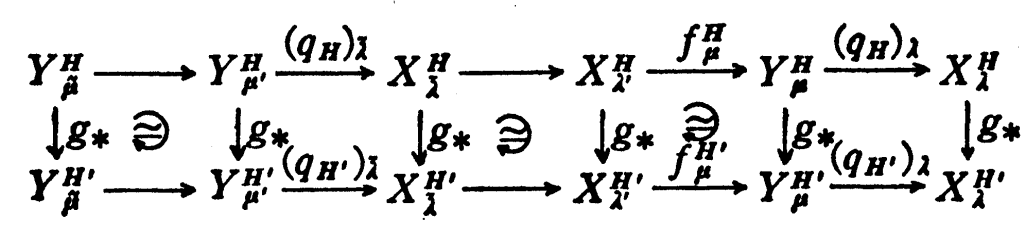

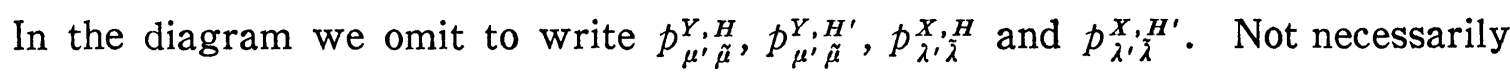
$g_{*}\left(q_{H}\right)_{\lambda} \simeq\left(q_{H^{\prime}}\right)_{\lambda} g_{*}$ but we have $g_{*}\left(q_{H}\right)_{\lambda} p_{\mu \tilde{\mu}}^{Y, H} \simeq\left(q_{H^{\prime}}\right)_{\lambda} p_{\mu \tilde{\mu}}^{Y, H^{\prime}} g_{*}$, because $g_{*}\left(q_{H}\right)_{\lambda} p_{\mu \tilde{\mu}}^{Y, H}$

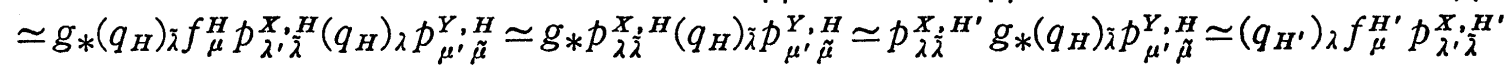
$\cdot g_{*}\left(q_{H}\right)_{\lambda} p_{\mu^{\prime} \tilde{\mu}}^{Y, H} \simeq\left(q_{H^{\prime}}\right)_{\lambda} g_{*} f_{\mu}^{H} p_{\lambda^{\prime}, \dot{\lambda}}^{X, H}\left(q_{H}\right)_{\bar{\lambda}} p_{\mu^{\prime} \tilde{\mu}}^{Y, H} \simeq\left(q_{H^{\prime}}\right)_{\lambda} g_{*} p_{\mu \tilde{\mu}}^{Y, H} \simeq\left(q_{H^{\prime}}\right)_{\lambda} p_{\mu \tilde{\mu}}^{Y, H^{\prime}} g_{* \cdot} \quad$ This means that we may assume $g_{*}\left(q_{H}\right)_{\lambda} \simeq\left(q_{H^{\prime}}\right)_{\lambda} g_{*}$ for every $H, H^{\prime}$ and $g$ by retaking $\tilde{\mu}$ as $\mu(\lambda)$. By Lemma 4.2 we get a new $G$-map $q_{\lambda}: Y_{\mu(\lambda)} \rightarrow X_{\lambda}$ such that

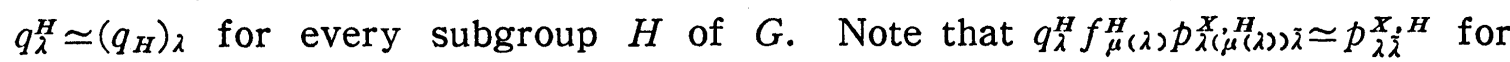
some $\tilde{\lambda} \geqq \lambda(\mu(\lambda))$ and every $H$. So, applying the same argument of Lemma 4.2, we can get a $G$-homotopy between $q_{\lambda} f_{\mu(\lambda)} p_{\lambda(\mu(\lambda)) \pi}^{X}$ and $p_{\lambda \tilde{\lambda}}^{X}$. Also, we have a $G$-homotopy between $f_{\mu} q_{\lambda(\mu)} p_{\mu(\lambda(\mu)) \tilde{\mu}}^{Y}$ and $p_{\mu \tilde{\mu}}^{Y}$ for some $\tilde{\mu} \geqq \mu(\lambda(\mu))$. q.e.d.

\section{Reserences}

[1] Antonian, S. A. and Mardešić, S., Equivariant shape, Fund. Math. 127 (1987), 213223.

[2] Borsuk, K., Theory of shape, Monografie Matematyczne 59, Polish Scientific Publishers, Warszawa, 1975.

[3] Dydak, J. and Segal, J., Shape theory, An introduction, Lecture Notes in Math. 688, Springer, Berlin, 1978.

[4] Mardešic, S. and Segal, J., Shape theory, The inverse system approach, NorthHolland Math. Library 26, Amsterdam, 1982.

[5] Matumoto, T., G-CW complexes and a theorem of J.H.C. Whitehead, J. Fac. Sci. Univ. Tokyo, IA 18 (1971), 109-125.

[6] - A complement to the theory of G-CW complexes, Japan. J. Math. 10 (1984), 353-374.

[7] Matumoto, T., Minami, N. and Sugawara, M., On the set of free homotopy classes and Brown's construction, Hiroshima Math. J. 14 (1984), 359-369.

[8] Morita, K., Theory of shape (in Japanese), Sûgaku 28 (1976), 335-347.

[9] Murayama, M., On G-ANR's and their G-homotopy types, Osaka J. Math. 20 (1983), 479-512.

[10] Pop, I., An equivariant shape theory, An. Stint. Univ. "A1. I. Cuza" Iaşi s. Ia Mat. 30-2 (1984), 53-67. 
[11] Smirnov, Yu. M., Shape theory of $G$-pairs, Uspekhi Mat. Nauk $40: 2$ (1985), 151$165=$ Russian Math. Surveys $40: 2$ (1985), 185-203.

[12] Whitehead, J.H. C., On $C^{1}$-complexes, Ann. Math. 41 (1940), 809-824.

Department of Mathematics

Faculty of Science

Hiroshima University

Hiroshima 730, Japan 